\title{
Modelling of the Thermo-Physical and Physical Properties for Solidification of Ni-based superalloys
}

\author{
N. Saunders, A. P. Miodownik and J.-Ph. Schillé \\ Sente Software Ltd., Surrey Technology Centre, The Surrey Research Park \\ Guildford, Surrey GU2 7YG, U. K.
}

\begin{abstract}
The thermo-physical and physical properties of the liquid and solid phases are critical components in the modelling of casting simulations. Such properties include the fraction solid transformed, enthalpy release, thermal conductivity, volume and density all as a function of temperature. Due to the difficulty in experimentally determining such properties at solidification temperatures, little information exists for multicomponent alloys. As part of the development of a new computer programme for modelling of materials properties (JMatPro), extensive work has been carried out on the development of sound, physically based models for these properties. Wide ranging results will presented for Ni-based alloys, which will include more detailed information concerning the phases formed during solidification and their composition and the density change of the liquid that intrinsically occurs during solidification due to its change in composition.
\end{abstract}

\section{INTRODUCTION}

Previous modelling work the phases formed on solidification, and their composition, by using thermodynamic modelling based on the

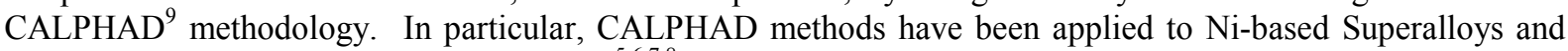
results checked in detail against experiment However, although useful in their own right, both for process modelling and modelling of microstructures, such calculations fall short of supplying physical property data for the phases, which is critical for successful simulation of solidification. At low temperatures, physical properties can be readily measured, although it may be a time-consuming and expensive procedure to obtain all relevant properties. Experimental measurement becomes far more problematical at high temperature and especially if the liquid phase is involved. To this end, it is highly desirable to calculate thermo-physical and physical properties over the complete relevant temperature range for as wide a range of alloys as possible. The present paper describes a methodology that extends the existing CALPHAD models to further calculate properties such as density, thermal conductivity, specific heat $\left(\mathrm{C}_{\mathrm{p}}\right)$, solidification shrinkage etc., and applies it for Ni-based multicomponent alloys. A significant advantage of the current method is that properties for each phase are calculated so fine detail can be obtained; for example the density change of the liquid during the solidification, which is governed both by an intrinsic change with temperature and by the composition changes that accompany solidification.

The current work forms part of the development of a more generalised software package (JMatPro) for the calculation of a wide range of materials properties. 10 A feature of the new programme is that great store has been placed on using models that, as far as possible, are based on sound physical principles rather than purely statistical methods. Thus, many of the shortcomings of methods such as regression analysis can be overcome. For example, the same model and model parameters are used for density calculations for all alloy types, whether it be for a commercially pure Al-alloy or a complex Ni-based superalloy.

The paper will discuss briefly the Scheil-Gulliver solidification model that is used to directly calculate phase amounts, $\mathrm{C}_{\mathrm{p}}$, enthalpy and latent heat of solidification. Details concerning the creation of a molar volume database that enables a variety of properties to be calculated, such as solidification shrinkage, density, thermal expansion coefficient, will then be presented. The calculation of thermal conductivity and modulus will also be discussed. Examples of the linking of the solidification models with the physical property calculations are made and properties calculated during solidification will be presented.

\section{THE SCHEIL-GULLIVER (SG) SOLIDIFICATION MODEL}

Recently the application of so-called 'Scheil-Gulliver' modelling via a thermodynamic calculation route has led to the ability to predict a number of critical thermo-physical properties for alloys Such calculations can be computationally very fast and used within solidification packages such as ProCAST ${ }^{5}$. The model assumes 
that solute diffusion in the solid phase is small enough to be considered negligible and that diffusion in the liquid is extremely fast, fast enough to assume that diffusion is complete. Such a process is quite simple to model using thermodynamic calculations based on the so-called CALPHAD method.

For equilibrium solidification described by the lever rule and with linear liquidus and solidus lines, the composition of the solid $\left(C_{S}\right)$ as a function of the fraction solid transformed $\left(f_{S}\right)$ is given by

$$
C_{s}=\frac{k C_{o}}{f_{s}(k-1)+1}
$$

where $k$ is the partition coefficient and $C_{o}$ is the composition of the original liquid alloy. This can be rearranged to give

$$
f_{s}=\left(\frac{1}{1-k}\right)\left(\frac{T_{L}-T}{T_{f}-T}\right)
$$

where $T$ is the temperature below the liquidus and $T_{L}$ and $T_{f}$ are, respectively, the equilibrium liquidus and solidus temperatures. A complementary limiting case to equilibrium solidification is to assume that solute diffusion in the solid phase is small enough to be considered negligible and that diffusion in the liquid is extremely fast, fast enough to assume that diffusion is complete. In this case eq. 1 can be re-written as

$$
C_{s}=k C_{o}\left(1-f_{s}\right)^{k-1}
$$

and eq. 2 as

$$
f_{s}=1-\left(\frac{T_{f}-T}{T_{f}-T_{L}}\right)^{\left[\frac{1}{k-1}\right]}
$$

The treatment above is the traditional derivation of the Scheil equation but it has quite severe restrictions when applied to multi-component alloys. It is not possible to derive this equation, using the same mathematical method, if the partition coefficient, $k$, is dependent on temperature and/or composition. The Scheil equation is applicable only to dendritic solidification and cannot, therefore, be applied to eutectic alloys which are commonplace for Ni-based alloys. Further, it cannot be used to predict the formation of intermetallics (e.g. the Laves phase) during solidification.

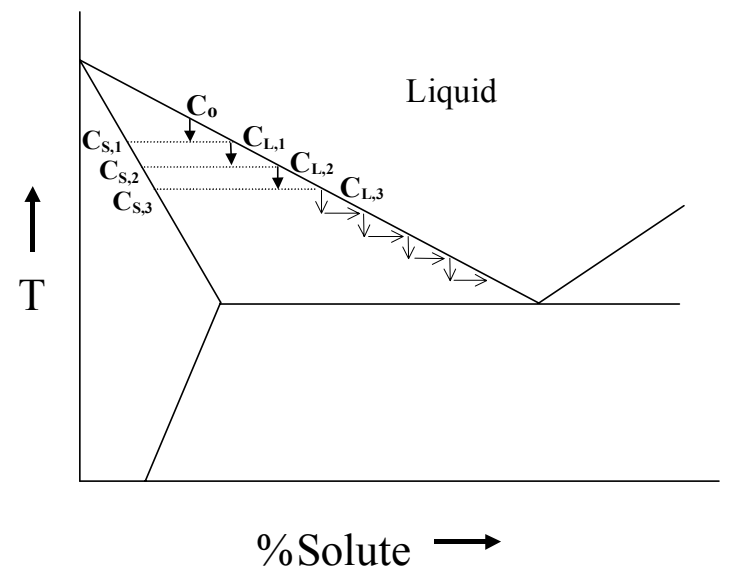

Figure 1. Schematic representation of solidification occurring under Scheil-Gulliver conditions
Using thermodynamic modelling, all of the above disadvantages can be overcome. The process that physically occurs during 'Scheil-type' solidification can be envisaged as follows (Fig.1). A liquid of composition $\mathrm{C}_{0}$, which in equilibrium would solidify as a complete solution, is cooled to a small amount below its liquidus. It precipitates out solid with a composition $\mathrm{C}_{\mathrm{S}, 1}$ and the liquid changes its composition to $\mathrm{C}_{\mathrm{L}, 1}$. However, on further cooling to the initial solid cannot change its composition due to lack of back diffusion and it is effectively 'isolated'. A local equilibrium is then set up where the liquid of composition $\mathrm{C}_{\mathrm{L}, 1}$ transforms to a liquid of composition $\mathrm{C}_{\mathrm{L}, 2}$ and a solid with composition $\mathrm{C}_{\mathrm{S}, 2}$, which is precipitated onto the original solid with composition $\mathrm{C}_{\mathrm{S}, 1}$. This process occurs again on cooling where the liquid of composition $\mathrm{C}_{\mathrm{L}, 2}$ transforms to a liquid of composition $\mathrm{C}_{\mathrm{L}, 3}$, and a solid with composition $\mathrm{C}_{\mathrm{S}, 3}$ grows on the existing solid. This process occurs continuously during cooling and when $\mathrm{k}<1$ leads to the solid phase becoming lean in solute in the center of the dendrite and the liquid becoming more and more enriched in solute as solidification proceeds. Eventually, the composition of the liquid will reach the eutectic composition and final solidification will occur via this reaction. 
Any appearance of secondary phases can be taken into account in this approach with the assumption that no back diffusion occurs in them. Therefore, all transformations can be accounted for, including the final eutectic solidification. The approach described here is based on an isothermal step process but, as the temperature step size becomes small, it provides results that are almost completely equivalent to that obtained from continuous cooling. A further and significant advantage of using a thermodynamic approach is that heat evolution during solidification is a product of the calculation. It is known that some back diffusion will occur but, in many cases, it is sufficiently limited such that the "Scheil-Gulliver" assumption leads to goodresults for much of the solidification range and can been used to obtain high quality input for casting simulations

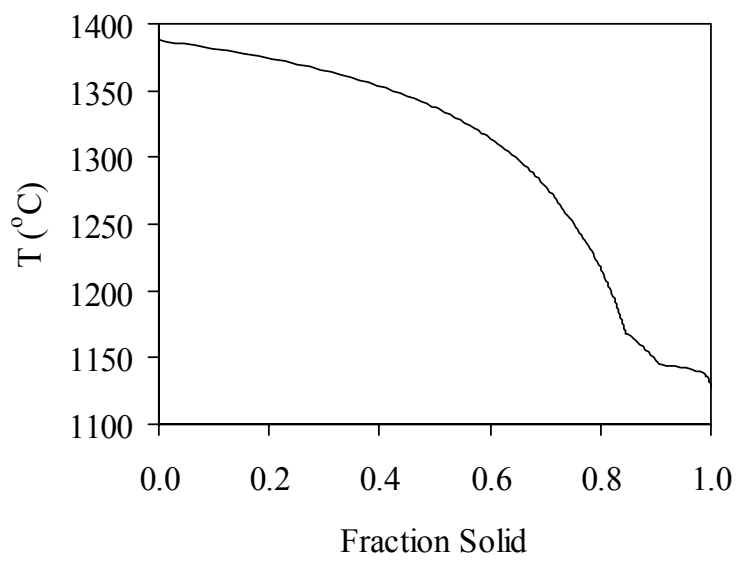

Figure 2. Calculated fraction solid vs. temperature plot for solidification of alloy 706.

Table I. Comparison between experimentally determined DTA results ${ }^{6}$ and a Scheil-Gulliver simulation for a 706 alloy

\begin{tabular}{cccccc}
\hline & Liquidus & $\begin{array}{c}\text { MC } \\
\text { start }\end{array}$ & $\begin{array}{c}\text { Laves } \\
\text { start }\end{array}$ & $\begin{array}{c}\eta \\
\text { start }\end{array}$ & $\begin{array}{c}\text { Solidifi- } \\
\text { cation end }\end{array}$ \\
\hline Centre (DTA) & 1381 & 1240 & 1164 & & \\
Edge (DTA) & 1388 & 1261 & & & \\
Calculated & 1388 & 1256 & 1168 & 1144 & 1128 \\
\hline
\end{tabular}

Figure 2 shows the calculated fraction vs temperature plot for alloy 706, while Table 1 shows the comparison between calculated and experimentally determined transformation temperatures. It is noted that $\eta$ is observed in solidified 706, though its formation temperature could not be discerned. At the completion of solidification, JMatPro retains information about the solid phases formed during solidification and extrapolates their properties below the solidus. Hence calculation of properties can be continued to low temperatures, enabling properties to be supplied to the whole mesh in a simulation (not just in the liquid and mushy zone). For example Figure 3 shows the calculated enthalpy from the casting temperature down to $600^{\circ} \mathrm{C}$ for alloy 706 . Fine detail can be obtained, for example, Figure 4 shows the segregation profiles in the as-solidified the Ni solid solution $(\gamma)\left(f_{s}=0\right.$ is the centre of a dendrite while $f_{s}=1$ indicates interdendritic region).

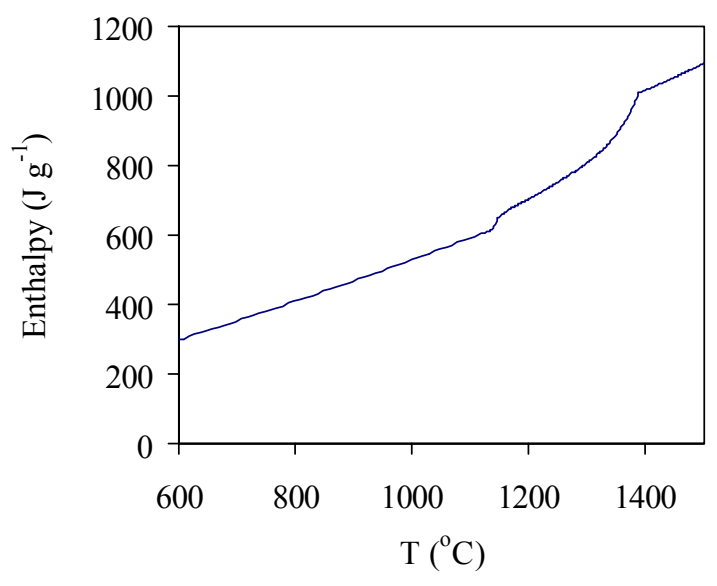

Figure 3. Enthalpy of alloy 706 during solidification.

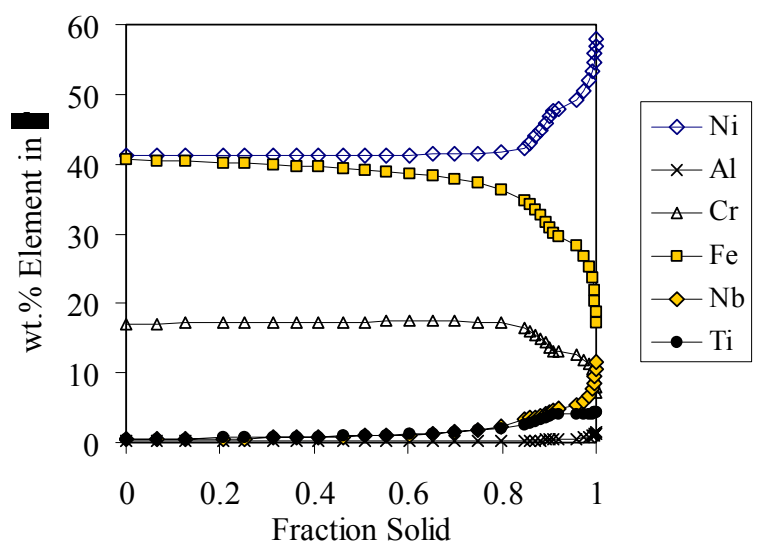

Figure 4. Calculated segregation patterns in the $\gamma$ phase after solidification of a 706 alloy. 


\section{PHYSICAL PROPERTY CALCULATIONS}

A major achievement of the JMatPro software project has been the development of an extensive molar volume database that can be linked to its thermodynamic calculation capability and hence provide volume data for the phases involved in the calculation. Presently, an extensive database of parameters exists for most of the major phases in Al-, Fe-, Mg-, Ni- and Ti-alloys and has been tested extensively in the solid state against lattice parameter measurements (both at room temperature and where available at high temperatures) and experimentally reported linear expansion data. Volume calculations are linked to the thermodynamic models such that, once a thermodynamic calculation is made, volume can be directly calculated.

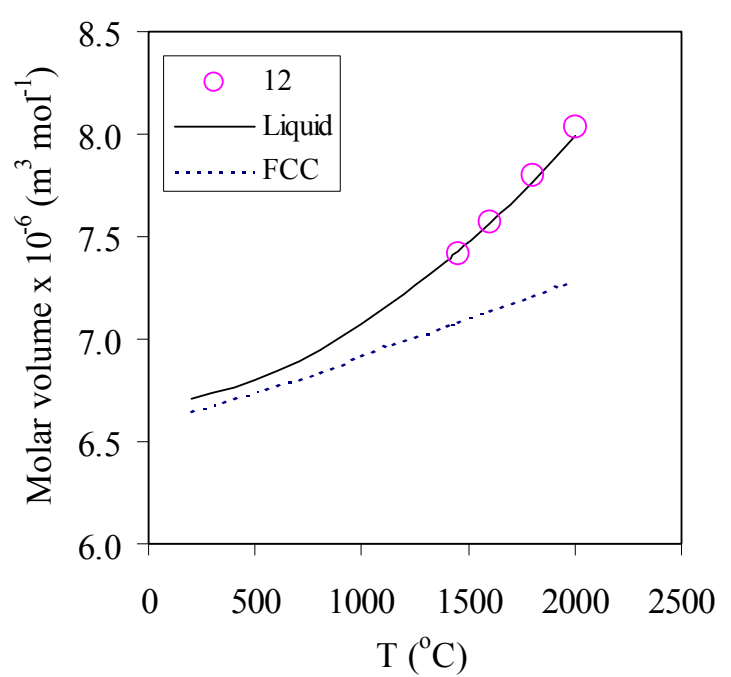

Figure 5. Calculated molar volumes for FCC and Liquid Ni.

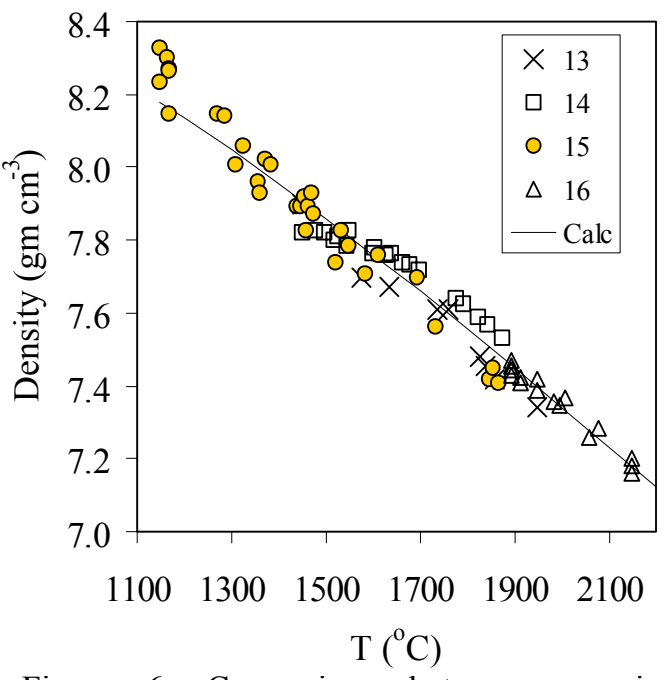

Figure 6. Comparison between experimentally determined and calculated density of liquid Ni.

The solid information can be directly linked to calculations for the liquid phase by comparison with measured liquid densities to provide volume changes on freezing, which are quite well documented for pure elements. Fig. 5 shows a plot of the molar volumes of the stable FCC and liquid phases in Ni. Also shown for comparison are values estimated by Sung et al.. 12 The densify of liquid Ni has been evaluated from experimentally measured values reported in the literature $13 \sqrt{14} \cdot 16$ (Figure 6) and combined with a model that extrapolates its properties to low temperatures.

The principle of the extrapolation method is based on the fact that the transition from the solid to liquid/amorphous state will be accompanied by an increase in volume associated with the larger volume of the liquid/amorphous phase in comparison to that of the crystalline structure. ${ }^{17}$ This means that the volume of the liquid should never reach that of the solid. We have also assumed that at a temperature close to the glass transition temperature $\mathrm{T}_{\mathrm{g}}$, (assumed $\sim 0.3 \mathrm{~T}_{\mathrm{m}}$ for metallic elements) the difference in volume will tend to reach a minimum and below $T_{g}$ the volume difference will be fairly constant. We have found that quite simple temperature functions give both a good fit to the temperature dependence of the density at high temperatures and a reasonable low temperature extrapolation based on the above principles.

We have considered the volume of the liquid and solid phases in multi-component systems as being represented by simple pair-wise mixture models, similar to those used to model thermodynamic excess functions in multi-component alloys.

$$
P=\sum_{i} x_{i} P_{i}^{o}+\sum_{i} \sum_{j>i} x_{i} x_{j} \sum_{v} \Omega_{v}\left(x_{i}-x_{j}\right)^{v}
$$

where, $P$ is the property of the phase, $P_{i}^{o}$ is the property of the phase in the pure element, $\Omega_{\mathrm{v}}$ is a binary interaction parameter, $x_{i}$ and $x_{j}$ are the mole fractions of elements $i$ and $j$ in the phase. It is also possible to include ternary or higher order effects where appropriate. In some alloys, the stable crystal structure of the element in solid solution may be different to that of the solid solution itself. For metallic solutes we have assumed the molar volume of the metastable state is the same as that for the stable structure. We have invariably found that if there is a deviation from "ideal mixing" a volume contraction is involved and that the magnitude of contraction is strongly governed by the magnitude of the thermodynamic interaction.

There have been few systematic investigations of alloying effects on the density of liquid alloys. An exception is the work of Lucas who directly measured the density of pure Fe and a series of binary Fe-alloys as 
a function of temperature and composition. Other than this, little information is available. Similarly, for multicomponent alloys, there are very few direct measurements available and, therefore, the authors are slightly wary of comparing calculations with estimates based on indirect correlation. However, it is instructive to compare calculated values with those reported in the open literature and Figure 7 shows such a comparison. The figure includes results for various alloys including $\mathrm{Al}$-, $\mathrm{Fe}$ - and Ni-based alloys to demonstrate the general effectiveness of the models used and to emphasise their generality of application. Two main sources of information have been used. (1) from the Aplurn solidification design centre, which are mainly estimations and (2) from literature citations by Sung et al. 2 . if density has been directly measured or estimated in some other way.

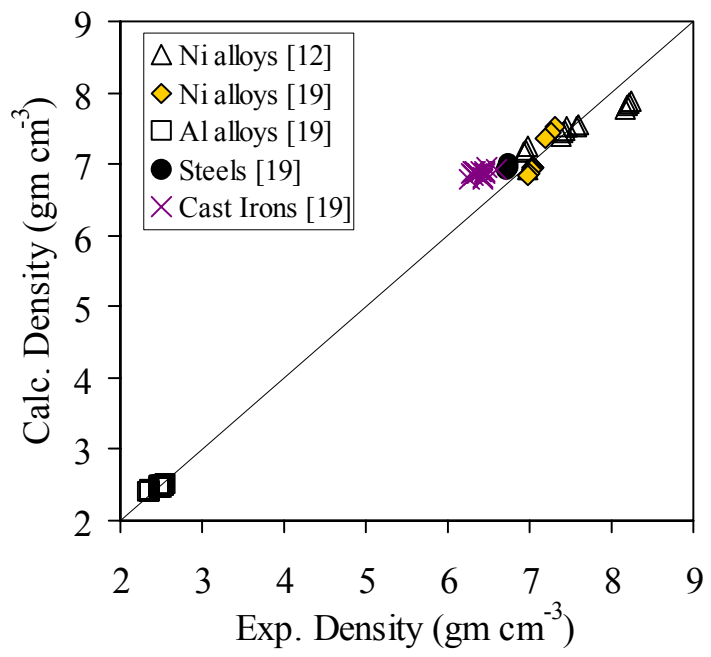

Figure 7. Comparison of calculated and reported densities for various multi-component alloys

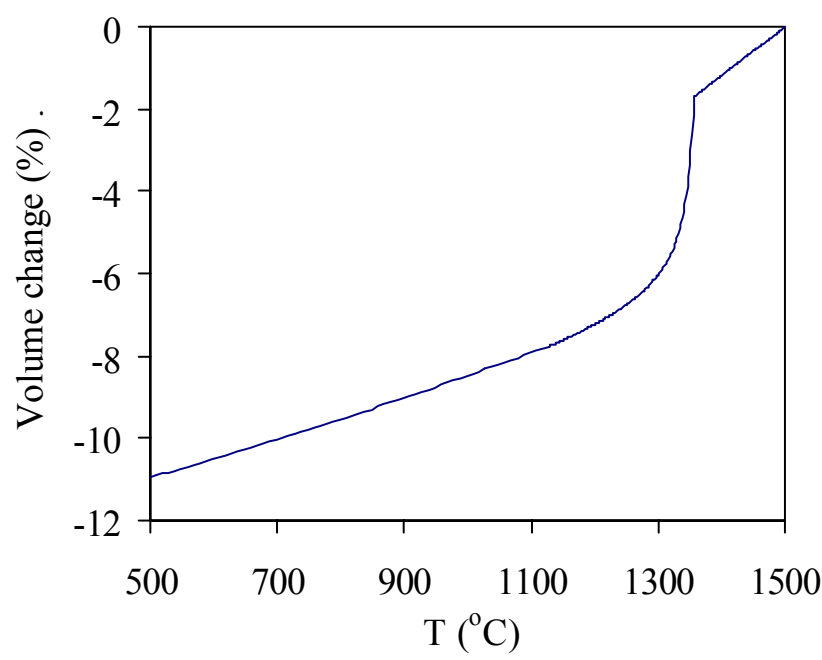

Figure 8. Calculated volume shrinkage for a Mar-M002 Ni-based superalloy during solidification

Overall, there is good agreement except for the Ni-based superalloy PWA1484 and cast irons where there is a clear discrepancy $(\sim 10 \%)$ between calculated densities and reported values. For the case of PHA 1484 the present result is rather similar to that found by Sung et al. necessary for this alloy. For the case of cast irons the Auburn group provide onty estimated values. Based on the direct measurements of Lucas ${ }^{8}$ for $\mathrm{Fe}, \mathrm{Fe}-\mathrm{C}$ and $\mathrm{Fe}-\mathrm{Si}$ alloys, all as a function of temperature, it is difficult to see that such low reported values for the density of liquid cast irons are accurate. In this case, we believe the current calculations provide answers that are more consistent with actual behaviour.

It is now possible to combine the $\mathrm{SG}$ model with the volume database to make calculations for solidification of various alloys. Fig. 8 shows the calculated shrinkage for a Mar-M002 Ni-based superalloy on casting. It should be noted that there are two parts to the shrinkage. There is the natural shrinkage that occurs on cooling of the liquid and solid phases and also the shrinkage that occurs due to the liquid to solid transformation itself. Note that for this case, and subsequent solidification calculations, properties are also calculated below the solidus so that the shrinkage occurring in the solid state can be accounted for.

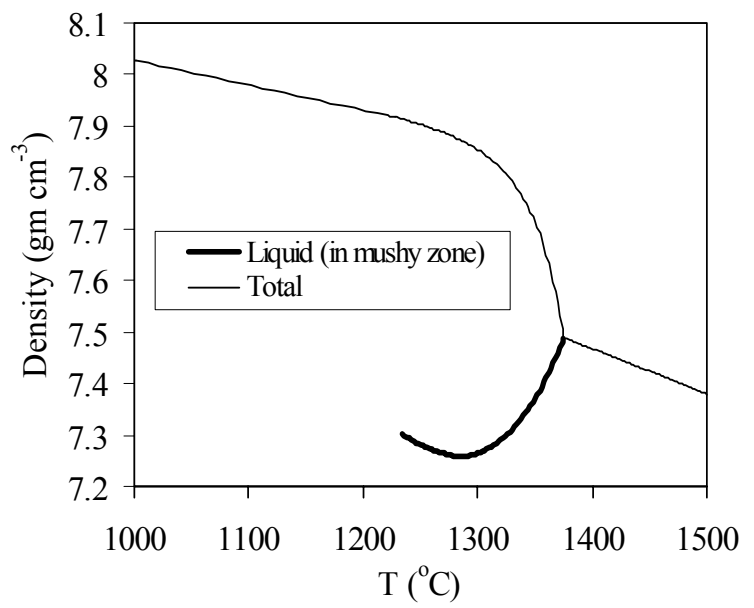

Figure 9. Calculated density of a SRR99 single crystal superalloy during solidification. Bold line shows density of the liquid in the mushy zone
Fig.9 shows a plot of density vs. temperature for a SRR99 single crystal Ni-based superalloy and, because we calculate the density for each phase, we have also shown the density of the liquid in the mushy zone. In this case, the heavy element Ta segregates to the liquid. $\mathrm{W}$ is depleted and additionally the light elements $\mathrm{Al}$ and $\mathrm{Ti}$ partition to the liquid. This combination of partitioning leads to the density inversion. It is not possible to directly measure the density of the liquid in the mushy zone and it is of particular interest because a density inversion is an important factor in determining susceptibility to freckle formation 20 . Generally speaking the density of the liquid in the mushy zone is important for other reasons, for example in modelling of macrosegregation and various defects. The ability to self-consistently calculate this property at will is a potentially powerful tool in achieving true predictive modelling of defects. 
Another important physical property, particularly for casting simulations, is the thermal conductivity. The calculation of thermal conductivity is more problematical in that it is a more complex physical phenomenon. There are sharp changes on alloying in the solid state and the behaviour in terms of mixing models is more difficult to match. On alloying, a "bath tub" shape is often seen, where the thermal conductivity falls sharply in the dilute range and then forms a fairly flat plateau in the concentrated region. 21 The current mixing models have been able to match this behaviour to a reasonable extent and extensive validation of solid state multicomponent alloys shows good results, see for example ref 10 for Ti-alloys.

Because of the dearth of information concerning the thermal conductivity of binary liquid alloys we cannot directly assess coefficients for alloying effects in the liquid. However, information does exist for thermal conductivity in the liquid state in pure elements and we have evaluated parameters using this information a a first basis. An invariable observation arising from the current assessment work is that to obtain the usually observed result in metals, i.e. that the thermal conductivity of the liquid is less than that of the liquid, the interaction coefficient for the liquid phase needed in eq.5 is either zero or only slightly negative. 24 We have therefore evaluated interaction terms based on this principle.

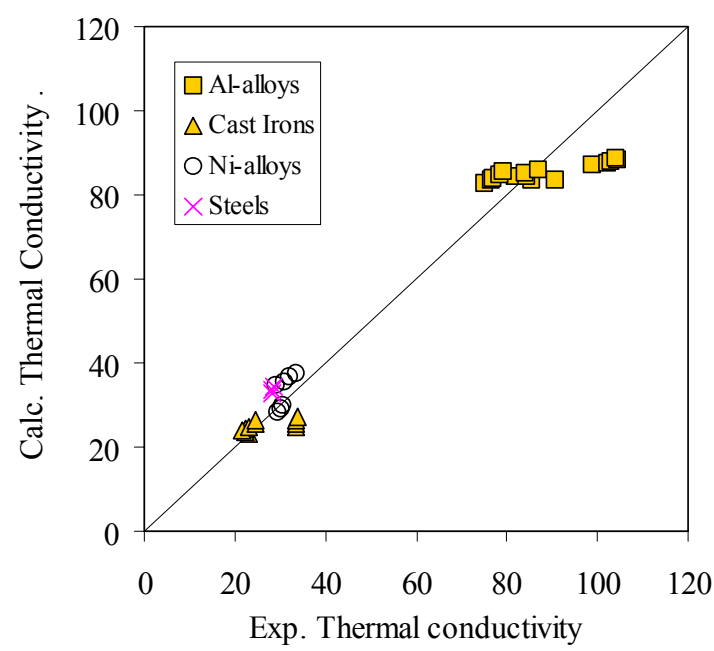

Figure 10. Comparison between calculated and experimental $^{19}$ thermal conductivities $\left(\mathrm{W} \mathrm{m}^{-1} \mathrm{~K}^{-1}\right)$ in multi-component alloys

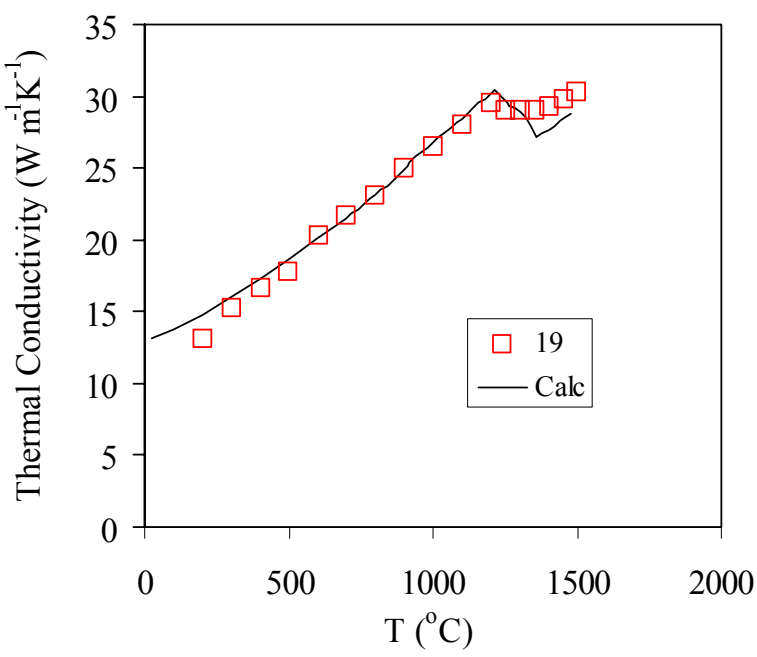

Figure 11. Comparison between calculated and

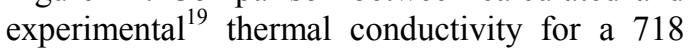
Ni-based superalloy (on heating).

In general, where results are available for multi-component liquid alloys good agreement is usually found. Fig.10 shows comparison with results from the Auburn University Centre for materials measurements for various cast irons, steels, Al-alloys and Ni-base superalloys. The agreement is rather good, except for a $201 \mathrm{Al}-$ alloy. In this case we rather trust the calculated values because one might generally expect the thermal conductivity to decrease when alloy elements are added in dilute solution, whereas to match experiment in A201, whose main alloying addition is $\sim 2 \mathrm{at} \% \mathrm{Cu}$, would require a quite sharp increase in thermal conductivity on alloying. An example of calculated thermal conducfixity on heating into the liquid state for a 718 Ni-based superalloys is shown in Fig.11 with experimental results 9 .

An interesting extension of work previously reported for solid state applications it is to include Young's, shear and bulk moduli and Poisson's ratio in the mushy state. Moduli calculations in the solid state, in combination with the volume calculations, are immediately useful for modelling of residual stresses in castings. However, when considering liquid metal processing and casting, the properties of the liquid need to be considered when modelling the mechanical properties in the semi-solid state. The position is such that the solid phases, which have finite shear and Young's moduli, co-exist with the liquid phase where these values are zero. When phases have such widely differing properties, it is well understood that a simple rule of mixtures should not be applied. More generalised, non-ideal mixture models are required. JMatPro uses a model for the physical properties of multi-phase mixtures after Fan et al.. ${ }^{5.66}$ The approach allows for effects such as amount of phase, contiguity, anisotropy as well morphology to be considered. The model originally considered two-phase materials but has been extended in JMatPro to include multi-phase alloys.

Figures 12 and 13 show, respectively, the fraction solid vs. temperature and the calculated shear modulus in the mushy zone of a Hastelloy $\mathrm{C}$ Ni-based superalloy. If a simple rule of mixtures is used, then the shear modulus plot would closely mirror the fraction solid plot, which is shown by the dashed line in Fig, 13 . However, this would not be expected in practice. In the current model, while the fraction solid curve increases sharply just below the liquidus, the shear modulus remains close to zero for the first $20-30 \%$ of transformation, 
's

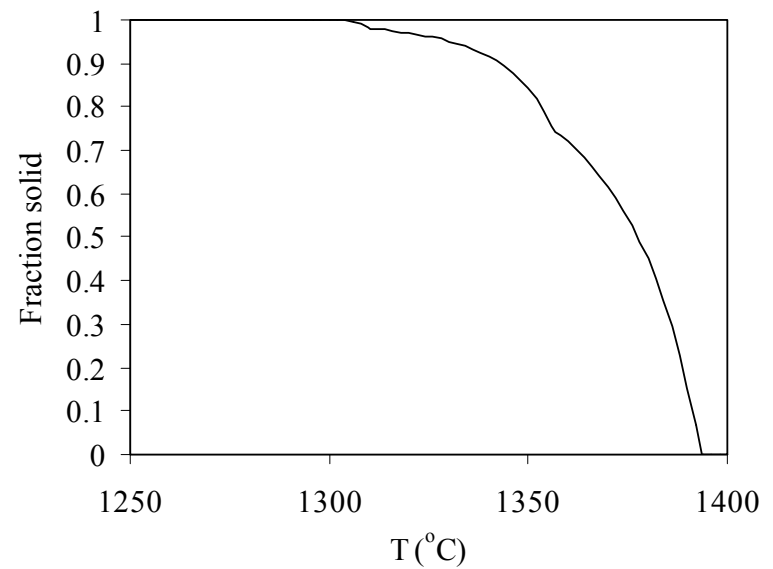

Figure 12. Calculated fraction solid vs. temperature plot for a Hastelloy C Ni-based superalloy.

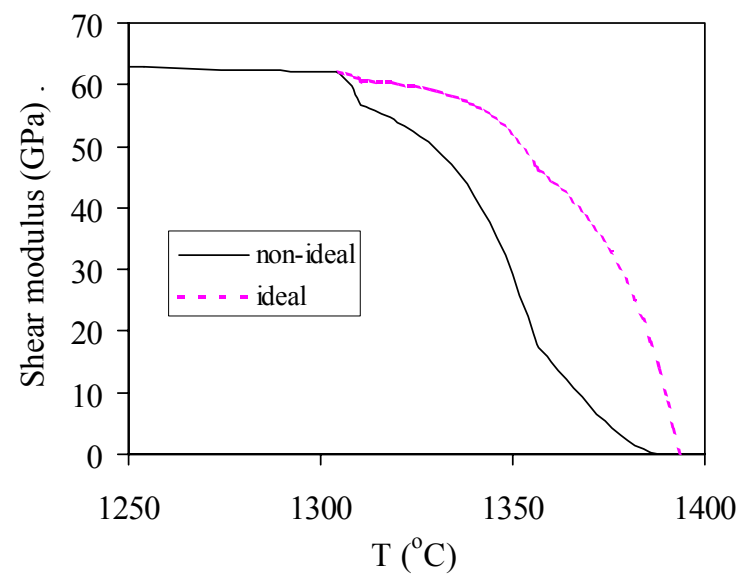

Figure 13. Calculated shear modulus for a Hastelloy C Ni-based superalloy during solidification, showing the effect of ideal and non-ideal rules of mixing.

which would provide a reasonable answer if the dendrite network becomes interconnected above $20-30 \%$. The current model assumes the mixture is isotropic and randomly distributed and that a small continuous fraction of solid exists in the very early stages of solidification. This scenario may not be ideally suited to solidification and knowledge of when the dendrite network becomes continuous should be an input. However, the purpose of the exercise is not to demonstrate accuracy of the current microstructural assumptions, but to demonstrate that microstructure sensitive, non-ideal rules of mixing can be considered and which can be tailored for specific cases of mushy zone morphology if required.

\section{SUMMARY AND CONCLUSIONS}

Models have been developed for the calculation of thermo-physical and physical properties of metallic alloys at solidification temperatures. Comparison with reported experimental behaviour is generally very good. Where significant discrepancies occur clear questions arise as to the validity of the experimental result. The present paper describes calculations for a wide range of thermo-physical and physical properties for Ni-based superalloys including fraction solid, enthalpy, density, shrinkage, thermal conductivity and modulus. Using known relationships between various properties it is also possible to expand on these properties to to provide an almost complete set of parameters that can be used for most types of modelling of casting and solidification. Work is currently undergoing to model viscosity to complete the comprehensive set of properties already available. A further, significant advantage of the approach described here, is that it is possible to obtain important properties for each phase individually. For example, the density of the liquid phase during the solidification process is automatically calculated.

\section{REFERENCES}

1. N. Saunders, Solidification Processing 1997, J. Beech and H. Jones, Eds., Univ. Sheffield, Sheffield, $1997,362$.

2. N. Saunders, Materials Science Forum, 217-222, 1996, 667.

3. N. Saunders, Light Metals 1997, R. Huglen, Ed., TMS, Warrendale, PA, 1997, 911.

4. R. A. Harding and N. Saunders, Trans. American Foundryman's Society, 105, 1997, 451.

5. W.J. Boettinger, U.R. Kattner, S.R. Coriell, Y.A. Chang and B.A Mueller, Modelling of Casting, Welding and Advanced Solidification Processes, VII, M. Cross et al., Eds., TMS, Warrendale, PA, 1995, 649.

6. B. A. Boutwell, R. G. Thompson, N. Saunders, S. K. Mannan, and J. J. deBarbadillo, Superalloys 718 , 625, 706 and Various Derivatives, E. A. Loria, Ed., TMS, Warrendale, PA, 1996, 99.

7. U. Grafe, D. Ma, A. Engstrom and S. G. Fries, Modelling of Casting, Welding and Advanced Solidification Processes VIII, B.G. Thomas and C. Beckermann, Eds., TMS, Warrendale, PA, 1998, 227.

8. M. S. A. Karunarate, D. C. Cox, P. Carter and R. C. Reed, Superalloys 2000, K. A. Green, T.M. Pollock and R.D. Kissinger, eds., TMS, Warrendale, PA, 2000, 263.

9. N. Saunders and A. P. Miodownik, CALPHAD - Calculation of Phase Diagrams, Pergamon Materials Series vol.1, R. W. Cahn, Ed., Elsevier Science, Oxford, 1998

10. N. Saunders, X. Li, A. P. Miodownik and J.-Ph. Schillé., Materials Design Approaches and Experiences, J.-C. Shao, M. Fahrmann and T. M. Pollock, Eds., TMS, Warrendale, PA, 2001, 185 
11. J. A. Spittle, S. G. R. Brown and M. Al Meshhedani, Numerical Methods in Thermal Analysis IX,

R. W. Lewis and P. Durbetaki, Eds., Pineridge Press, Swansea, 1995, 455.

12. P. K. Sung, D. R. Poirrer and E. McBride, Mat. Sci. Eng., A231, 1997, 189.

13. A. D. Kirshenbaum and J. A. Cahill, Trans. AIME, 224, 1962, 816; Trans ASM, 56, 1963, 281

14. A. M. Samarin and A. A. Bertman, Dokl. Akad . Nauk, SSSR, 155, 1964, 323.

15. S. Y. Shiraishi and R. G. Ward, Canadian Met. Quarterly, 3, 1964, 117

16. T. Saito and Y. Sakuma, J. Japan Inst. Metals, 31, 1967, 1140

17. J. L. Finney, The Structure of Non-Crystalline Materials, ed. P.H. Gaskell (London: Taylor \& Francis, 1977), 35

18. L. D. Lucas, Mem. Sci. Rev. Met., 61, 1964, 97.

19. Data downloaded from web address http://metalcasting.auburn.edu/data/data.html

20. P. Auburtin, S. L. Cockcroft and A. Mitchell, Superalloys 1996, R. D. Kissinger et al., Eds., TMS, Warrendale, PA, 1996, 443

21. J. B. Austin The Flow of Heat in Metals, ASM, Cleveland, OH, 1942.

22. T. Iida and R. L. Guthrie, The Physical Properties of Liquid Metals, Clarendon Press, Oxford, 1988).

23. K. C. Mills, B. J. Monaghan and B. J. Keene, Int. Mater. Rev., 41, 1996, 209.

24. N. Saunders, X. Li. A. P. Miodownik and J.-Ph. Schillé, Modelling of Casting, Welding and Advanced Solidification Processes X, D. Stefanescu, J. A. Warren, M. R. Jolly and M. J. M. Krane, Eds., TMS, Warrendale, PA, 2003, 669.

25. Z. Fan, P. Tsakiropoulos, and A. P. Miodownik, J. Mater. Sci., 29, 1994, 141.

26. Z. Fan, Phil. Mag. A, 73, 1996, 1663. 